\title{
Controlling and Synchronization of a New Lorenz-like System
}

\author{
Qiguo Yao ${ }^{\mathrm{a}, *}$, Yuxiang Su', Lili Li ${ }^{\mathrm{c}}$ \\ School of Naval Architecture \& Mechanical-electrical Engineering, Zhejiang Ocean University, Zhoushan, \\ Zhejiang, 316022, China \\ ayaoqiguo@163.com, bsuyuxiang82@163.com, clilili@zjou.edu.cn \\ *corresponding author
}

Keywords: Chaos, Lyapunov Function, Backstepping Control, Adaptive Synchronization

\begin{abstract}
Using the back stepping design method, a simple controller is formulated to control the system to its unstable equilibrium $(0,0,0)$, any point of the form $(0,0,1+b+p(1-p))(0<p<1)$ in phase space, and force the first state variable to trace arbitrary given function $r(t)$. Suppose one parameter was unknown, a unidirectional coupled scheme and a parameter update role are proposed to assure the new Lorenz-like system to globally synchronize with a copy of itself. Numerical simulations using Matlab were presented to show the feasibility of our design.
\end{abstract}

\section{Introduction}

Chaos, as a very interesting nonlinear phenomenon, has been intensively studied over the past decades. Since Lorenz found the first classical chaotic attractor in 1963, many new attractors of a three-dimensional continuous-time autonomous system were coined. For example, Rossler system, Chua's circuit, Chen system etc. In 2002, Jinhu Lü and Guanrong Chen reported a new chaotic system called Lü system, which satisfies condition $a_{12} a_{21}=0$, and bridges the gap between the Lorenz and Chen systems[1][2]. In the same year, a unified chaotic system was created that connects Chen chaotic system to the Lorenz chaotic system through the Lü chaotic system. Li presented a new Lorenz-like system where the sign of the crucial condition $a_{12} a_{21}$ is only determined by parameter $b[3]$. We will investigate the control and synchronization problem of this new Lorenz-like system.

The control and synchronization of chaotic systems are extensively studied fields in non-linear dynamics that were introduced in 1990 by Ott et a1 using a scheme known as OGY closed-loop method, and Pecora and Carroll using a scheme called APD method respectively.

In this paper, we investigate the control problem of this new Lorenz-like system by using the backstepping design method, we formulate a simple controller to control the system to its unstable equilibrium $(0,0,0)$, any point of the form $(0,0,1+b+p(1-p))(0<p<1)$ in phase space and force the first state variable to track arbitrary given function $r(t)$. The adaptive design is used to deal with the synchronization problem of the new Lorenz-like system.

\section{The New Lorenz-like System}

The new Lorenz-like chaotic system[4] is:

$$
\left\{\begin{array}{l}
\dot{x}=a(y-x) \\
\dot{y}=a b x-a x z \\
\dot{z}=x y-c z
\end{array}\right.
$$

Where $(x, y, z)^{T} \in R^{3}$ is the state variables of the system, $a, b$ and $c$ are parameters. It is easy to verify that system (1) is globally, uniformly, and asymptotically stable about its zero equilibrium if $a>0, b<0, c>0$. It is dissipative when $a+c<0$. The system (1) would satisfy one of the following 
cases via the crucial condition set by Vanecek : $a_{12} a_{21}>0$ when $b>0 ; a_{12} a_{21}<0$ when $b<0$, and $a_{12} a_{21}=0$ when $b=0$. This shows that the sign of the crucial condition $a_{12} a_{21}$ is determined only by parameter $b$. When $a \neq 0$ and $b>0$, the equilibrium $(0,0,0)$ is unstable.

\section{Controlling and Synchronization of the System}

\subsection{Controlling the System to Any Point.}

In order to control the new Lorenz-like system (1), we add a control input $u_{1}$ to the third equation of system (1) and rewrite the controlled as

$$
\left\{\begin{array}{l}
\dot{x}=a(y-x) \\
\dot{y}=a b x-a x z \\
\dot{z}=x y-c z+u_{1}
\end{array}\right.
$$

Our objective is to find a control law $u_{1}$ to stabilize the state of the controlled system (2) at a bounded point. Starting from the first equation, a stabilizing function $\alpha_{1}(x)$ has to be designed for the virtual control $y$ in order to make the derivative of $V_{1}=x^{2} / 2$, i.e., $\dot{V}_{1}(x)=-a x^{2}+a x y$ be negative definite. Let $\alpha_{1}(x)=p x$ and define an error variable as

$$
\bar{y}=y-\alpha_{1}(x)
$$

We obtained the $(x, \bar{y})$-subsystem

$$
\left\{\begin{array}{l}
\dot{x}=a \bar{y}-a(1-p) x) \\
\dot{\bar{y}}=a b x-a x z-a p \bar{y}+a p(1-p) x
\end{array}\right.
$$

We construct a Lyapunov function for the subsystem as follows:

$$
V_{2}(x, \bar{y})=V_{1}(x)+\frac{1}{2} \bar{y}^{2}
$$

Calculating the time derivative of $V_{2}(x, \bar{y})$ along system (4), we have $\dot{V}_{2}(x, \bar{y})=-a(1-p) x^{2}-a p \bar{y}^{2}-a x \bar{y}[z-1-b-p(1-p)]$

We can choose: $z=\alpha_{2}(x, \bar{y})=1+b+p(1-p)$, then $\dot{V}_{2}(x, \bar{y})=-a(1-p) x^{2}-a p \bar{y}^{2}<0$ if $0<p<1$. Similarly, allowing

$$
\bar{z}=z-\alpha_{2}(x, \bar{y})
$$

We get the following system in the $(x, \bar{y}, \bar{z})$ coordinates

$$
\left\{\begin{array}{l}
\dot{x}=a \bar{y}-a(1-p) x) \\
\dot{\bar{y}}=a b x-a x z-a p \bar{y}+a p(1-p) x \\
\dot{\bar{z}}=x \bar{y}+p x^{2}-c \bar{z}-c[1+b+p(1-p)]+u_{1}
\end{array}\right.
$$

We can construct a Lyapunov function as follows:

$$
V_{3}(x, \bar{y}, \bar{z})=V_{2}(x, \bar{y})+\frac{a}{2} \bar{z}^{2}
$$

Calculating the time derivative of $V_{3}(x, \bar{y}, \bar{z})$ along system (6), we have

$$
\dot{V}_{3}(x, \bar{y}, \bar{z})=-a(1-p) x^{2}-a p \bar{y}^{2}-a c \bar{z}^{2}+a \bar{z}\left[p x^{2}-c\left(1+b+p-p^{2}\right)+u_{1}\right]
$$


If we choose the control input $u_{1}$ as

$$
u_{1}=c\left(1+b+p-p^{2}\right)-p x^{2}
$$

Then $\dot{V}_{3}(x, \bar{y}, \bar{z})$ is negative, therefore system (6) has been stabilized at the origin point $(0,0,0)$ according to the Lyapunov stability theory. Thus the system (2) has been stabilized at the point $(0,0,1+b+p(1-p))(0<p<1)$ if we note the transformation (3) and (5).

\subsection{Controlling the System to Origin Point $(0,0,0)$.}

In order to control the new system to the unstable equilibrium point $(0,0,0)$, we add a controller $u_{2}$ to the second equation of system (1) and rewrite the controlled system as [5][6]:

$$
\left\{\begin{array}{l}
\dot{x}=a(y-x) \\
\dot{y}=a b x-a x z+u_{2} \\
\dot{z}=x y-c z
\end{array}\right.
$$

For the virtual control $y$, we design a stabilizing function $\alpha_{1}(x)$ to make the derivative of $V_{1}(x)=x^{2} / 2, \dot{V}_{1}(x)=-a z^{2}+a x y$, be negative definite as $y=\alpha_{1}(x)$, let $\alpha_{1}(x)=0$ and define an error variable:

$$
\bar{y}=y-\alpha_{1}(x)
$$

We obtain the following $(x, \bar{y})$-subsystem:

$$
\left\{\begin{array}{l}
\dot{x}=a(\bar{y}-x) \\
\dot{\bar{y}}=a b x-a x z+u_{2}
\end{array}\right.
$$

We construct a Lyapunov function for the subsystem as follows:

$$
V_{2}(x, \bar{y})=V_{1}(x)+\frac{1}{2} \bar{y}^{2}
$$

Calculating the time derivative of $V_{2}(x, \bar{y})$ along system (10), we have;

$$
\dot{V}_{2}(x, \bar{y})=-a x^{2}+\bar{y}\left(a x+a b x-a x z+u_{2}\right)
$$

In order to make (11) be negative definite, we choose:

$$
u_{2}=-a x(1+b-z)-\bar{y}
$$

Then the controller (12) guarantee the equilibrium $(0,0)$ of the subsystem (10) to be asymptotically stable by Lyapunov stability theory.

\subsection{Tracking Any Desired Function.}

We will construct a control law $u_{3}$ so that a scalar output $x(t)$ of the new Lorenz-like system can track any desired function $r(t)$. The controlled new Lorenz-like system [7] is:

$$
\left\{\begin{array}{l}
\dot{x}=a(y-x) \\
\dot{y}=a b x-a x z+u_{3} \\
\dot{z}=x y-c z
\end{array}\right.
$$

The controller $u_{3}$ is to be designed. Let $\bar{x}(t)=x(t)-r(t)$, define a function $U_{1}=\bar{x}^{2} / 2$ and calculate its time derivative along the orbit of the controlled system (13) we get

$$
\dot{U}_{1}=(x-r)[a(y-x)-\dot{r}]
$$


In order to make $\dot{U}_{1}$ to be negative definite, we choose the virtual control $\mathrm{y}$ as $y=\alpha(x)=x+(r+\dot{r}-x) / a$ and define an error variable $\bar{y}=y-\alpha(x)$. We obtain the $(x, \bar{y})$ subsystem.

$$
\left\{\begin{array}{l}
\dot{\bar{x}}=a(\bar{y}-\bar{x})-a r+a \alpha-\dot{r} \\
\dot{\bar{y}}=a b \bar{x}+a b r-a \bar{x} z-a r z-\dot{\alpha}+u_{3}
\end{array}\right.
$$

Let $U_{2}=U_{1}+\bar{y}^{2} / 2$, we will get

$$
\dot{U}_{2}=-a \bar{x}^{2}-a r \bar{x}+\alpha a \bar{x}-\dot{r} \bar{x}+\bar{y}\left(a \bar{x}+a b \bar{x}-a \bar{x} z-a r z+a b r-\dot{\alpha}+u_{3}\right)
$$

By choosing the control law $u_{3}$ as follows:

$$
u_{3}=a x z-a b x-2 a x-2 y+a y+2 x+\frac{\ddot{r}+2 \dot{r}+r-x}{a}+a r
$$

Then the origin $(0,0)$ of system(14) is asymptotically stable, and this implies that $\lim _{t \rightarrow \infty} \bar{x}(t)=0$.

\subsection{Adaptive Synchronization of New Lorenz-like System.}

In order to observe synchronization of the new system, we assume parameter $a$ is unknown and the new Lorenz-like system (1) is the drive system [8]:

$$
\left\{\begin{array}{l}
\dot{x}=a(y-x) \\
\dot{y}=a b x-a x z \\
\dot{z}=x y-c z
\end{array}\right.
$$

And the response system is:

$$
\left\{\begin{array}{l}
\dot{x}_{1}=a\left(y_{1}-x_{1}\right)+u_{1} \\
\dot{y}_{1}=a b x_{1}-a x_{1} z_{1}+u_{2} \\
\dot{z}_{1}=x_{1} y_{1}-c z_{1}+u_{3}
\end{array}\right.
$$

Where $u_{1}, u_{2}, u_{3}$ are three control functions to be designed. In order to determine the control functions to realize synchronization between system (17) and (18), we subtract (17) from (18) and get the error system:

$$
\left\{\begin{array}{l}
\dot{e}_{1}=a\left(e_{2}-e_{1}\right)+u_{1} \\
\dot{e}_{2}=a b e_{1}-a e_{1} z_{1}-a x e_{3}+u_{2} \\
\dot{e}_{3}=e_{1} y_{1}+x e_{2}-c e_{3}+u_{3}
\end{array}\right.
$$

Where $e_{1}=x_{1}-x, e_{2}=y_{1}-y, e_{3}=z_{1}-z$.Our goal is to find proper control functions $u_{i}(i=1,2,3)$ and a parameter update rule such that system (18) globally synchronizes with system (17), i.e. $\lim _{t \rightarrow \infty}\|e(t)\|=0$ where $e=\left[e_{1}, e_{2}, e_{3}\right]^{T}$.

We propose the following adaptive control law for system (18).

$$
\left\{\begin{array}{l}
u_{1}=\bar{a}\left(e_{1}-e_{2}-b e_{2}+e_{2} z_{1}\right)-e_{3} y-e_{1} \\
u_{2}=\bar{a} x e_{3}-x_{1} e_{3}-e_{2} \\
u_{3}=0
\end{array}\right.
$$

And the parameter update rule for the unknown parameter $a$ is:

$$
\dot{\bar{a}}=e_{1}\left(e_{2}-e_{1}\right)+e_{2}\left(b e_{1}-e_{1} z_{1}-x e_{3}\right)+(a-\bar{a})
$$


Where $\bar{a}$ is estimated value of $a$.

Theorem: For any initial conditions, the response system (18) and the drive system (17) are globally synchronize by adaptive control law (20) and parameter update rule (21).

Proof: Substitute the control law (20) to (19) we get the error dynamics as follows.

$$
\left\{\begin{array}{l}
\dot{e}_{1}=\tilde{a}\left(e_{2}-e_{1}\right)+\bar{a}\left(-b e_{2}+e_{2} z_{1}\right)-e_{3} y-e_{1} \\
\dot{e}_{2}=\tilde{a}\left(b e_{1}-e_{1} z_{1}-x e_{3}\right)+\bar{a}\left(b e_{1}-e_{1} z_{1}\right)-x_{1} e_{3}-e_{2} \\
\dot{e}_{3}=e_{1} y_{1}+x e_{2}-c e_{3}
\end{array}\right.
$$

Where $\tilde{a}=a-\bar{a}$. Consider the following Lyapunov function:

$$
V=\frac{1}{2}\left(e_{1}^{2}+e_{2}^{2}+e_{3}^{2}\right)+\frac{1}{2} \tilde{a}^{2}
$$

The time derivative of $V$ along the solution of error system (22) gives that

$$
\dot{V}=e^{T} e+\tilde{a} \dot{\bar{a}}=-e_{1}^{2}-e_{2}^{2}-c e_{3}^{2}-\tilde{a}^{2}
$$

Thus origin $(0,0,0)$ of system(22) is globally asymptotic stable, i.e. $\lim _{t \rightarrow \infty}\|e(t)\|=0, \forall e(0) \in R^{3}$.

Therefore, the state variables $x_{1}, y_{1}, z_{1}$ of the response system (19) and the state variables $x, y, z$ of the drive system (17) are globally synchronized asymptotically.

\section{Numerical Simulations}

\subsection{Numerical Simulation for Control the New Lorenz-like System.}

In this section, numerical simulations are carried out using Matlab. The four-oder Runge-Kutta method is used to solve two systems of differential (2) and (7) with a time step size 0.01 . We select the parameters of the Lorenz-like system (1) as $a=5, b=4, c=2$, which is the typical chaotic case. The initial states of the controlled the new system (8) is, $x_{0}(0)=20 y_{0}(0)=-20, z_{0}(0)=15$. Fig. 1 shows that the new system can be stabilized with the control law $u_{2}$ to the bounded point.
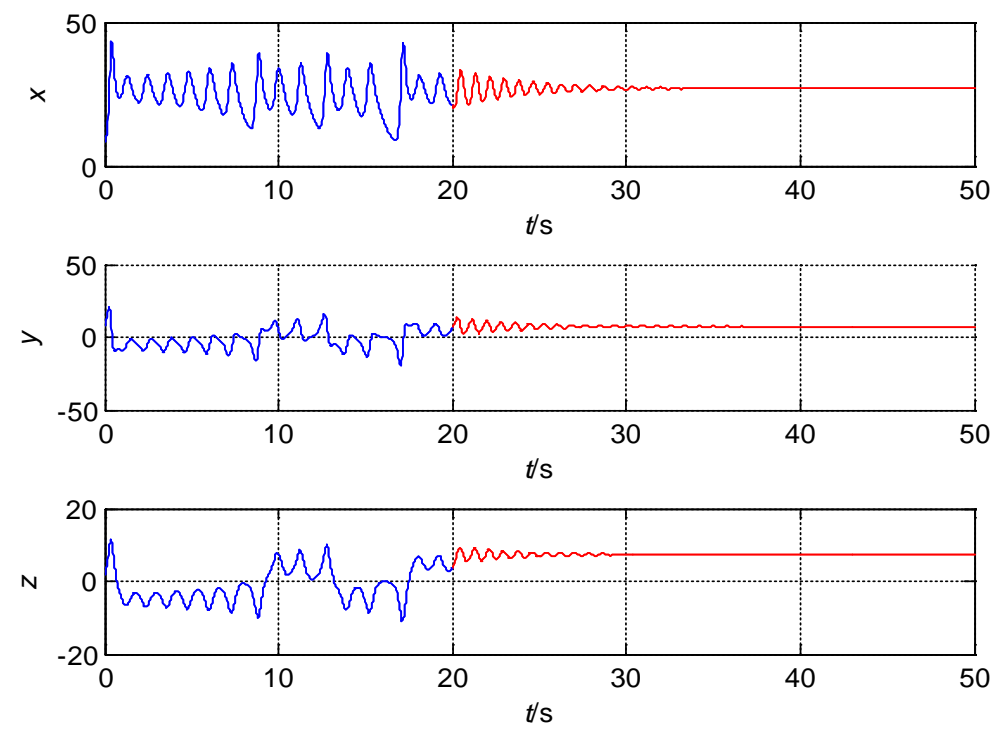

Fig. 1 The wave form of the the states $x, y, z$ for the controlled svstem (8) with the control indut 


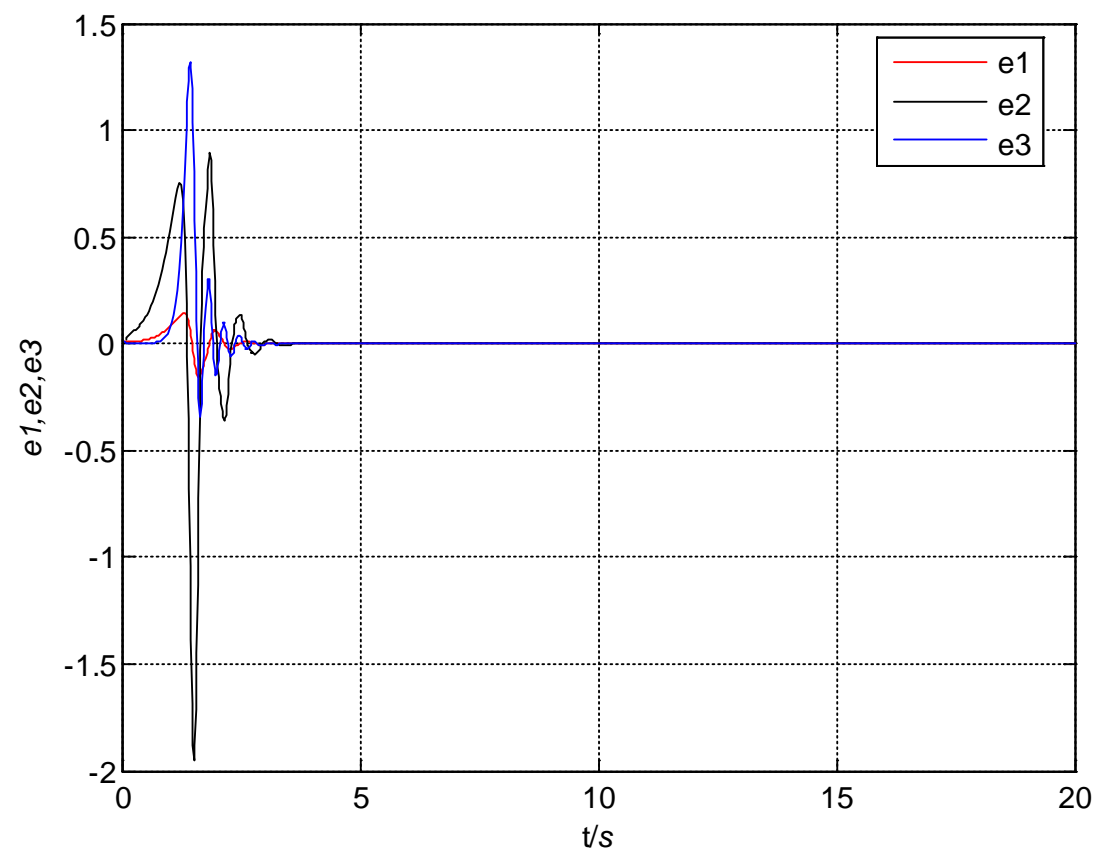

Fig.2 The wave form of the synchronization errors $\left(e_{1}, e_{2}, e_{3}\right)$ of system(17) and system(18)

\subsection{Numerical Simulation for Synchronization.}

Now we demonstrate the effectiveness of the proposed method for synchronization of the drive system (17) and the response system (18). In the numerical simulation, the fourth-order Runge-Kutta integration method is used to solve the systems of differential equations (17)-(19) and (21) with time step size 0.001 . We assume that the initial values of the drive and response systems are $(x(0), y(0), z(0))=(-1,-1,-1)$ and $\left(x_{1}(0), y_{1}(0), z_{1}(0)\right)=(2,3,4)$, respectively, then the initial error of system $(19)$ is $\left(e_{1}(0), e_{2}(0), e_{3}(0)\right)=(3,4,5)$. We choose $b=4, c=2$ and the reference value of the unknown parameter a of system (1) is chosen as $a=5$, the initial estimated parameter $\bar{a}(0)=10$. Fig.2 displays the synchronization errors between systems (17) and (18). Obviously, the synchronization errors converge asymptotically to zero. Furthermore, the estimated values of unknown parameters converge to $a=5$ when $t \rightarrow \infty$. In this case, we need only one controller to realize controlling and synchronization between chaotic systems. Moreover, we use backstepping design to enable stabilization of chaotic motion to steady state as well as tracking of any design trajectory to be achieved in a systematic way.

\section{Conclusion}

In this paper, by using the backstepping design method we formulate a simple controller to control the system to its unstable equilibrium $(0,0,0)$, any point of the form $(0,0,1+b+p(1-p))(0<p<1)$ in phase space and force the first state variable to trace arbitrary given function $r(t)$. Assume one parameter is unknown, we proposed an adaptive control law and a parameter update role to assure the new Lorenz-like system to globally synchronize with a copy of itself, which based on Lyapunov stability theory. The digital results obtained shows that the backing control is effective to steady state as well as tracking of any desired trajectory to be achieved in a systematic way.

\section{Acknowledgements}

This research was supported by Zhejiang Provincial Natural Science Foundation of China under Grant No. LQ18E070004 and Grant No. LQ15F030007. 


\section{References}

[1] Jinhu Lü , Guanrong Chen. A new chaotic attractor coined[J]. International Journal of Bifurcation and Chaos, 2002, 12(3):659-661.

[2] Jinhu Lü, Guanrong Chen,Daizhan Chen. Bridge the gap between the Lorenz system and the Chen system[J]. International Journal of Bifurcation and Chaos, 2002, 12(12):2917-2926.

[3] Xianfeng Li, Yandong Chu, Jiangang Zhang. Nonlinear dynamics and circuit implementation for a new Lorenz-like attractor[J]. Chaos, Solitons \& Fractals, 2009, 41(5):2360-2370.

[4] Shihua Chen, Limin Zhao, Jie Liu. Parameter identification and synchronization of an uncertain Chen chaotic system via adaptive control[J]. Chinese Physics, 2002, 11(6):543-546.

[5] H. Salarieh, M. Shahrokhi. Adaptive synchronization of two different chaotic systems with time varying unknown parameters[J]. Chaos, Solitons \& fractals, 2008, 37(1):125-136.

[6] Jinhu Lü, Suochun Zhang. Controlling Chen's chaotic attractor using backstepping design based on parameters identification[J]. Physics Letters A, 2001, 286(2-3):148-152.

[7] Xiaohui Tan, Jiye Zhang, Yiren Yang. Synchronizing chaotic systems using backatepping design[J]. Chaos, Solitons \& Fractals, 2003, 16(1):37-45.

[8] M.T. Yassen. Controlling, synchronization and tracking chaotic Liu system using active backstepping design[J]. Physics Letters A, 2007, 360(4-5):582-587. 